\title{
Progress on the control of hepatitis B infection through immunisation
}

\author{
M A Kane
}

\begin{abstract}
Hepatitis $B$ virus (HBV) infection and its sequelae, one of the major diseases of mankind, is now a disease preventable by vaccine. During the last few years, the price of these vaccines has fallen to the point where widespread use in infant immunisation programmes is feasible. This paper reports the great progress that has been made in the control of $\mathrm{HBV}$ infection and describes the targets that must be met if HBV control on a global basis is to be achieved.
\end{abstract}

(Gut 1993; supplement: S10-S12)

The hepatitis B virus (HBV) has infected more than 2000 million persons alive today, and 350000000 persons are chronically infected carriers of the virus, at high risk of death from chronic active hepatitis, cirrhosis, and primary hepatocellular carcinoma (Fig 1). Primary hepatocellular carcinoma is the number one or two cause of death by cancer in men in much of Asia, the Pacific Basin, and sub Saharan Africa. Each year, it is estimated that from 1000000 to 1500000 people die from the acute and chronic sequelae of $\mathrm{HBV}$ infection, making it one of the main causes of morbidity and death in man.

$\mathrm{HBV}$ infection is preventable with vaccines which have been available for a decade, and which have been used in more than 100000000 people with an outstanding record of safety and efficacy. The vaccines were initially too costly to consider for mass immunisation programmes and were primarily used to immunise health workers in industrialised countries. Over the past five years, the price of the vaccines for public sector programmes in developing countries has fallen dramatically, allowing public health officials in some industrialised and developing countries to begin to use this vaccine as a routine childhood immunogen.

\section{Immunisation programmes}

Both plasma derived $\mathrm{HBV}$ vaccines, the first to be developed, and DNA recombinant vaccines, are now widely available and are safe and effective. DNA recombinant vaccines are produced by manufacturers in the USA, Belgium, France, and Japan. Plasma derived vaccines are produced in South Korea, China, and Japan. Most people still receive the plasma derived product, and China alone will produce up to 45 million doses during 1992. In developing countries, plasma derived vaccines are somewhat less expensive, varying from $\$ 0.59$ to $\$ 1.25$ per dose. The cost of DNA recombinant vaccines in developing countries varies from $\$ 1.25$ to $\$ 2.00$ per dose. In industrialised countries, the price on the private market is quite high, with three adult doses selling for approximately $\$ 120 \cdot 00$. The US government, however, purchases paediatric doses of vaccine for public sector use for less

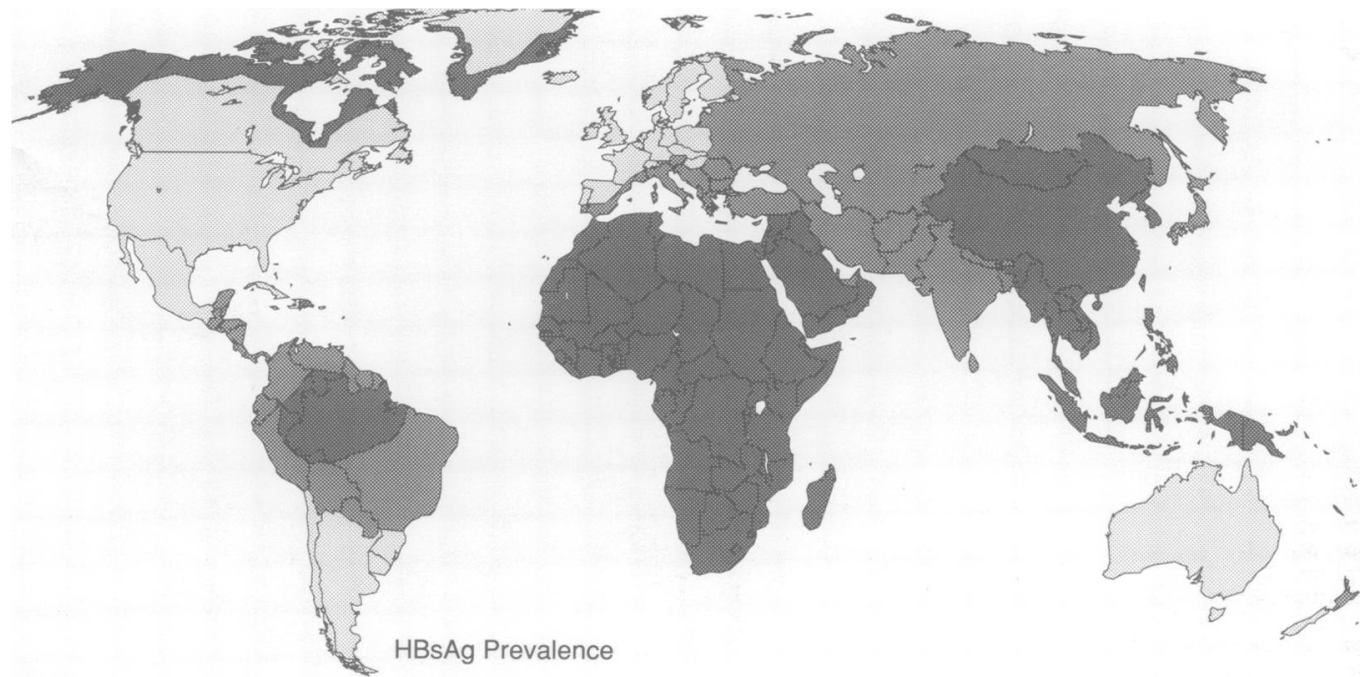


than $\$ 7.00$ per dose which is in the price range for other paediatric vaccines in the USA.

When HBV vaccine became available in 1982 , strategies for its use were based on the epidemiological pattern of $\mathrm{HBV}$ infection in the country of use. For areas with high and intermediate endemicity of $\mathrm{HBV}$ infection, where the predominant modes of transmission are child to child and mother to child (perinatal) transmission, the correct strategy was thought to be universal immunisation of infants. In areas of 'low' endemicity, such as western Europe and North America, most HBV infection occurred in certain 'high risk' groups defined by lifestyle or occupation, and vaccine was targeted to these groups. The "high risk' group strategy failed to make a significant impact on the incidence of HBV infection, and now universal immunisation of infants (and in some cases adolescents) is being increasingly recognised as necessary, even in areas of 'low' endemicity.

There are a number of reasons for the failure of the 'high risk' group strategy in countries of low endemicity. About $80-90 \%$ of vaccine was given to one high risk group, health care workers, which represents fewer than $5 \%$ of cases in the USA, and a minority of cases in European countries. Although health care workers should receive this vaccine as a matter of highest priority, we cannot control the disease on a population basis with this strategy alone. Other groups who contribute much more to $\mathrm{HBV}$ infection, such as intravenous drug users, homosexually active men, and heterosexually active people with multiple partners, proved much more difficult to identify. Many patients with HBV infection do not belong to any identified risk group and cannot be targeted in selected programmes. Finally, most countries have substantial populations of ethnic minorities, refugees, and immigrants from areas of high endemicity who continue to maintain endemic transmission of the virus. In the USA, the incidence of $\mathrm{HBV}$ continued to increase for several years after the introduction of the vaccine. Among 'low' prevalence countries, immunisation practices advisory committees in Canada, the USA, Italy, and New Zealand have now recommended universal infant immunisation with $\mathrm{HBV}$ vaccine.

The importance of $\mathrm{HBV}$ infection and the feasibility of its control with vaccine has led to the Global Advisory Group of the Expanded Programme on Immunisation to call for introduction of HBV vaccine into the Expanded Programme of Immunisation several times since 1987. In 1991, the Global Advisory Group set targets for introduction of HBV vaccine into National Immunisation Programmes. It called for countries with a carrier prevalence of $8 \%$ or more to have national programmes in place by 1995 , and for all other countries to have programmes in place by 1997 . These targets were endorsed by the World Health Assembly in May 1992 (Fig 2).

By the end of 1992, about 41 countries will have a national policy to give $\mathrm{HBV}$ vaccine to all newborns as part of their national immunisation programmes. Infants in these countries represent about $32 \%$ of births, but more than $50 \%$ of $\mathrm{HBV}$ carriers in the world. Some of these countries are phasing in the HBV immunisation over several years. Many other countries are planning to introduce HBV vaccine in the near future, or want to introduce the vaccine but cannot afford to obtain it.

\section{Cost effectiveness of infant immunisation} The use of HBV vaccine is following economical paths instead of epidemiological ones. The more prosperous countries in Asia and the Middle East are adopting HBV immunisation, while the poorer countries, especially in sub Saharan Africa, cannot obtain the vaccine despite a high carrier prevalence $(8-15 \%)$ and a great burden of disease. Donor agencies which provide most of the vaccine to countries in Africa have not yet been willing to supply $\mathrm{HBV}$ vaccine.
Figure $2 H B V$ vaccine policy in 1992: infant

immunisation. Footnote The design
employed and the presentation of material on presentation of material on this map do not imply the expression of any opinion whatsoever on the part of the World Health Organisation concerning the legal status of any country, territory, city or area of its authorities, or concerning the delimitation of its frontiers or boundaries.

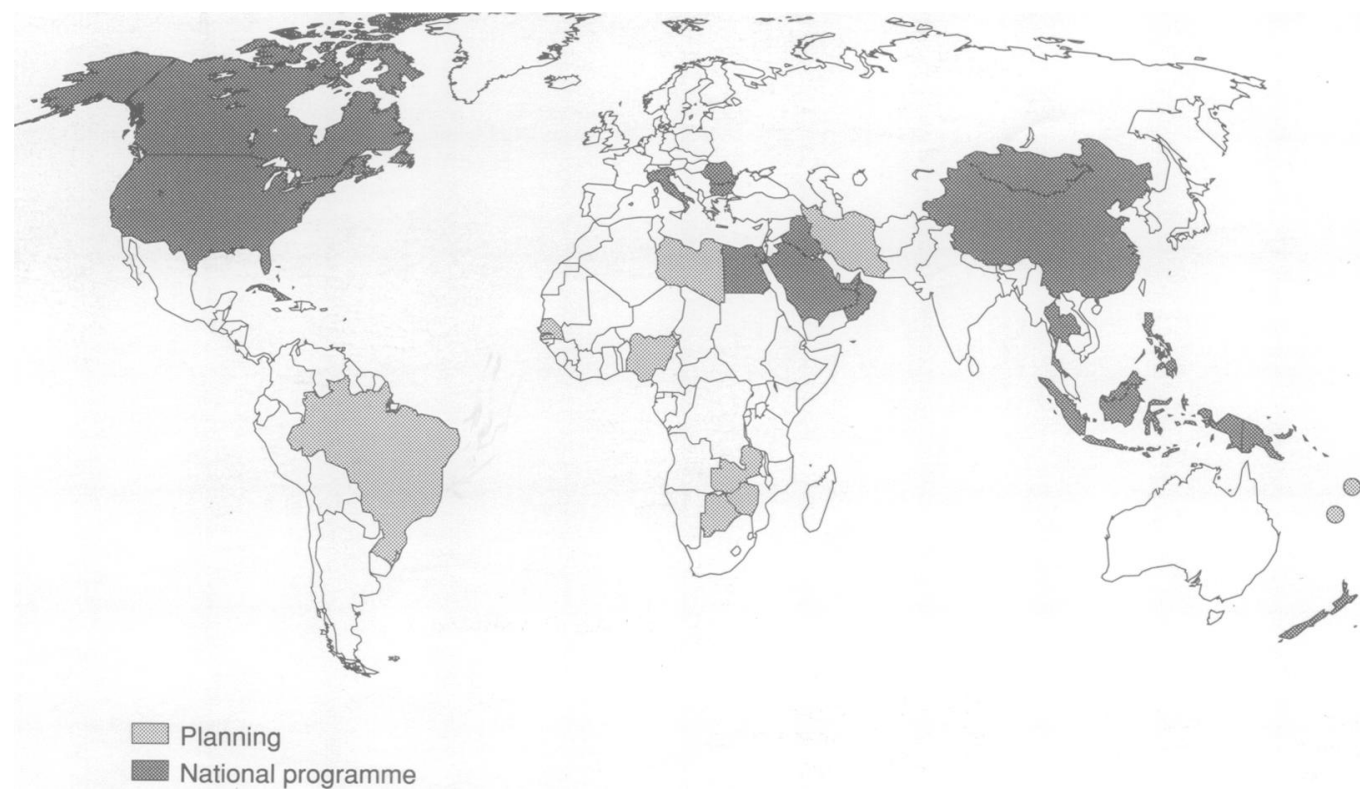


HBV immunisation is the most cost effective tool available to control HBV infection. The efficacy of vaccine in preventing the carrier state is between 90 and $95 \%$. HBV vaccine is now available for $\$ 0.59-2.00$ per paediatric dose in developing countries. Three doses are needed and it costs an additional $\$ 1.30$ per child to deliver the vaccine. At one dollar per paediatric dose, the cost effectiveness of adding HBV immunisation to the national immunisation programme in a developing country is approximately $\$ 30-40$ per carrier prevented.

In contrast, treatment with interferon after the carrier state develops is of marginal effectiveness, especially in adult carriers in countries of high endemicity who were most likely to have been infected as small children. Treatment costs thousands of dollars, making it unavailable to all but a tiny fraction of the world's carriers. Although interferon treatment may lead to early seroconversion from hepatitis $\mathrm{B}$ e antigen (HBeAg) to anti-HBe, it should be remembered that most hepatitis $B$ surface antigen (HBsAg) carriers who develop primary hepatocellular carcinoma have anti-HBe at the time the tumour develops, and it is not yet clear whether interferon treatment will influence progression to cancer in HBV carriers. Once primary hepatocellular carcinoma develops, there is no effective treatment and death occurs within one to three months after symptomatic presentation in developing countries. In industrialised countries, it may be possible to prolong life with surgery, chemotherapy, and other expensive interventions, but the death rate still exceeds $90 \%$.

Attempts to screen HBsAg carriers and patients with cirrhosis for alpha fetoprotein or by using ultrasound, or both, have been partially successful in identifying early primary hepatocellular carcinoma tumours which may be resectable or treatable with ethanol injection. The efficacy and cost effectiveness of this approach to identify and treat early primary hepatocellular carcinoma cases needs further study.

\section{Summary and conclusions}

With the adoption of universal infant immunisation with $\mathrm{HBV}$ vaccine in 41 countries, and with set targets for introduction by $1995 / 1997$, it is fair to say that enormous progress has been made, and that the stage has been set for global control of HBV infection. The successful attainment of the targets will depend primarily on what happens in sub Saharan Africa. If donor support for vaccine procurement can be found, and if routine EPI vaccine coverage can be increased to the high levels found in other regions of the world, we will be able to achieve control of $\mathrm{HBV}$ infection on a global basis. 